\title{
Estimativa de Parte Relevante da Fronteira da Região de Estabilidade usando Função Energia Generalizada
}

\author{
Yuri C. S. Ribeiro ${ }^{1}$
}

Escola de Engenharia de São Carlos, USP, São Carlos, SP

Departamento de Pesquisa e Desenvolvimento, Tecumseh do Brasil Ltda., São Carlos, SP

Luís F. C. Alberto ${ }^{2}$

Escola de Engenharia de São Carlos, USP, São Carlos, SP

Resumo. Neste trabalho, apresentamos um método para estimar parte relevante da fronteira da região de estabilidade associada a um conjunto atrativo de um sistema não linear através do uso de uma função energia generalizada. A teoria desenvolvida permite concluir que a estimativa obtida é sempre conservadora, em um certo sentido. Apresentamos um algoritmo conceitual e sua aplicação é ilustrada por meio de um exemplo.

Palavras-chave. Método CUEP, Funções Energia Generalizadas, Região de Estabilidade, Sistemas Elétricos de Potência, Estabilidade Transitória.

\section{Introdução}

Considere o sistema de equações diferenciais

$$
\dot{x}(t)=f(x(t)),
$$

onde $\dot{x}(t)$ denota a derivada de $x(t)$ com respeito a variável $t$ e $f: \mathbb{R}^{n} \rightarrow \mathbb{R}^{n}$ é uma aplicação de classe $\mathcal{C}^{1}$. Denotaremos por $t \mapsto \Phi\left(t, x_{0}\right)$, a única solução de (1) satisfazendo $\Phi\left(0, x_{0}\right)=x_{0}$.

Um conjunto atrativo $\sigma$ é um conjunto fechado e invariante que possui uma vizinhança aberta $U$ tal que, para todo $x_{0} \in U$, temos $\Phi\left(t, x_{0}\right) \rightarrow \sigma$ quando $t \rightarrow \infty$, ou seja, a distância entre $\Phi\left(t, x_{0}\right)$ e $\sigma$ tende para zero à medida que $t \rightarrow \infty$.

O conjunto $\omega$-limite de $x$, denotado por $\omega(x)$, é o conjunto de todos os pontos $p$ para os quais existe uma sequência de tempos $\left\{t_{n}\right\} \operatorname{com} t_{n} \rightarrow \infty$ e $\left|\Phi\left(t_{n}, x\right)-p\right| \rightarrow 0$ quando $n \rightarrow \infty$.

Se $\sigma$ é um conjunto atrativo, definimos a região de estabilidade de $\sigma$ por

$$
A(\sigma)=\left\{x \in \mathbb{R}^{n}: \omega(x) \subset \sigma\right\} .
$$

A região de estabilidade de conjuntos atrativos desempenha papel fundamental no estudo do comportamento assintótico de sistemas dinâmicos. Por exemplo, no estudo da

\footnotetext{
${ }^{1}$ yribeiro@usp.br

2lfcalberto@usp.br
} 
imunização de uma população com respeito a uma dada doença (ver [8]), em controle automático de vôo (ver [3]) ou no estudo de estabilidade transitória de sistemas elétricos de potência (ver [7]).

Agora, dado um número real $t_{s}>0$, considere um sistema regido por equações do tipo,

$$
\left\{\begin{array}{l}
\dot{x}=g(t, x) \\
x(0)=x_{0}
\end{array}, \text { onde } g(t, x)=\left\{\begin{array}{l}
\tilde{f}(x), t \in\left[0, t_{s}\right] \\
f(x), t>t_{s}
\end{array},\right.\right.
$$

onde $\tilde{f}$ e $f$ são funções de classe $\mathcal{C}^{1}$ de $\mathbb{R}^{n}$ nele próprio.

Este tipo de modelo surge, tipicamente, no estudo do impacto de determinadas perturbações na dinâmica de um dado sistema. O sistema se encontra no estado $x_{0}$ quando é submetido a uma perturbação de duração igual a $t_{s}$. Durante a perturbação, sua dinâmica é regida pelo campo de vetores $\tilde{f}$. Depois disso, a perturbação é removida e o sistema se comporta com dinâmica regida pela função $f$.

Por isso, os subsistemas $\dot{x}=\tilde{f}(x)$ e $\dot{x}=f(x)$ serão denominados, respectivamente, sistema perturbado e sistema pós-perturbação.

Suponha que a dinâmica do sistema pós-perturbação possua um conjunto atrativo $\sigma$ que corresponda a um comportamento desejável para o sistema em regime permanente. Dizemos que o sistema (2) suporta a perturbação, quando ele evolui para $\sigma$ após a remoção da perturbação. Para que isso ocorra, é necessário e suficiente que, no instante $t_{s}$, a solução do sistema perturbado $\dot{x}=\tilde{f}(x)$ esteja contida na região de estabilidade $A(\sigma)$ com respeito ao sistema pós-perturbação $\dot{x}=f(x)$.

A análise de estabilidade deste tipo de sistema consiste em determinar se o sistema suporta a perturbação com duração $t_{s}$. A pergunta que surge naturalmente é: como podemos determinar a máxima duração da perturbação para que o sistema ainda a suporte?

Esta pergunta pode ser respondida se pudermos detectar o instante em que a solução do sistema perturbado $\dot{x}=\tilde{f}(x)$ intersecta a fronteira da região de estabilidade $\partial A(\sigma)$ com respeito à dinâmica pós-perturbação. Para tanto, basta que consigamos uma estimativa para uma parte relevante de $\partial A(\sigma)$, ou seja, na direção da trajetória do sistema perturbado.

A necessidade de se obter tal estimativa, mais precisamente aquela que surge quando se estuda perturbações causadas por faltas em sistemas elétricos de potência, deu origem a um método chamado CUEP (ver [7]). Porém, o método CUEP está fundamentado na teoria das funções energia (FE) (ver [6]) e depende da existência de tal função para que possa ser aplicado ao sistema sob análise.

A existência de uma FE para um sistema tem implicações sobre o comportamento dinâmico de suas soluções. De fato, toda solução limitada converge para um ponto de equilíbrio quando o tempo tende para infinito. Consequentemente, sistemas que possuem órbitas periódicas ou soluções caóticas não admitem FE (ver [4]).

É neste contexto que o conceito de função energia generalizada foi introduzido em $[1,2]$. Embora funções energia generalizadas sejam mais fáceis de serem encontradas e possam ser admitidas por uma classe bastante geral de sistemas, é necessário encontrar resultados que deem suporte a métodos para a análise da estabilidade em sistemas que as admite.

Neste trabalho um método para estimar partes relevantes da fronteira da região de estabilidade inspirado no método CUEP é proposto para sistemas dinâmicos que admitem função energia generalizada. 


\subsection{Funções Energia Generalizadas}

Considere o sistema (1) e seja $V: \mathbb{R}^{n} \rightarrow \mathbb{R}$ uma função de classe $\mathcal{C}^{2}$. Defina o conjunto

$$
C=\left\{x \in \mathbb{R}^{n}: \nabla V(x) \cdot f(x) \geq 0\right\} .
$$

Em [1] foi introduzida a seguinte classe de funções auxiliares.

Definição 1.1. Uma função $V: \mathbb{R}^{n} \rightarrow \mathbb{R}$ é dita ser uma função de energia generalizada (FEG) para o sistema (1), quando as seguintes afirmações são verdadeiras,

G1. O conjunto $C$ é limitado;

G2. $O$ conjunto $C$ possui um número finito de componentes conexas;

G3. Para uma dada solução $t \mapsto \Phi\left(t, x_{0}\right)$ do sistema (1), se a função $t \mapsto V\left(\Phi\left(t, x_{0}\right)\right)$ é limitado para $t>0$, então a solução $\Phi\left(t, x_{0}\right)$ também é limitada para $t>0$.

A simples existência de uma FEG tem implicações importantes sobre o comportamento das trajetórias do sistema (ver [1]). Por exemplo, toda trajetória sobre a fronteira da região de estabilidade é limitada e converge para um conjunto que intersecta a fronteira do conjunto $C$ (ver Teorema 4.3 de [1]).

\section{A Parte Relevante da Fronteira da Região de Estabilidade}

Ao se obter uma estimativa para uma parte relevante da fronteira da região de estabilidade, é desejável que ela seja conservadora, ou seja, a solução do sistema perturbado deve intersectar a estimativa da fronteira da região de estabilidade antes de intersectar a fronteira da região de estabilidade verdadeira. Desta forma, a estimativa permite afirmar com certeza se o sistema suporta uma dada perturbação.

Definição 2.1. Considere o sistema (2). Ao menor tempo $t$ tal que $\Phi(t, x) \in \partial A(\sigma) e$ $\Phi(t+\varepsilon, x) \notin \overline{A(\sigma)}$ para $\varepsilon>0$ arbitrariamente pequeno, dá-se o nome de tempo crítico $e$ denota-se por $t_{c}$. Além disso, $\Phi\left(t_{c}, x\right)$ é chamado exit-point.

Para garantir a existência do tempo crítico e do exit-point, é necessário supor que o ponto $x_{0}$ pertença a $A(\sigma)$ e que a trajetória do sistema perturbado se afaste de $\sigma$ tanto quanto necessário para cruzar a fronteira de $A(\sigma)$. Ambas hipóteses são bastante razoáveis em diversas aplicações.

Dado um conjunto $B \subset \mathbb{R}^{n}$, definimos o conjunto

$$
\mathcal{W}^{s}(B)=\left\{x \in \mathbb{R}^{n}: \overline{\Phi^{+}(t, x)} \cap B \neq \varnothing\right\},
$$

onde $\Phi^{+}(t, x)$ denota a trajetória futura de $x$, ou seja, $\Phi^{+}(t, x)=\{\Phi(t, x), t \geq 0\}$.

Com a notação acima, podemos enunciar os seguintes resultados. 
Teorema 2.1. Considere que o sistema (1) possua um conjunto atrativo $\sigma$ e que admita uma FEG, denotada por $V: \mathbb{R}^{n} \rightarrow \mathbb{R}$. Tomando $\Lambda=\left\{i \in \mathbb{N}: C_{i} \cap \partial A(\sigma) \neq \varnothing\right\}$, tem-se

$$
\partial A(\sigma) \subset \bigcup_{i \in \Lambda} \mathcal{W}^{s}\left(C_{i}\right)
$$

A demonstração deste resultado segue diretamente do Teorema 4.3 de [1].

Teorema 2.2. Considere as mesmas hipóteses do Teorema 2.1 e seja $x \in \partial A(\sigma)$. Se $\Phi^{+}(x) \cap C=\varnothing$, então $\omega(x) \subset C_{i}$, para algum $i \in \Lambda=\left\{i \in \mathbb{N}: C_{i} \cap \partial A(\sigma) \neq \varnothing\right\}$.

Demonstração. Tome $x \in \partial A(\sigma)$. Pela Proposição 4.2 de [1], $\omega(x)$ é conjunto invariante não-vazio e conexo.

Por hipótese, $\Phi^{+}(x) \cap C=\varnothing$ portanto $t \mapsto V(\Phi(t, x))$ é uma função decrescente limitada por baixo (ver Proposição 4.1 de [1]). Seja $L \in \mathbb{R}$ o limite de $V(\Phi(t, x)$ ) quando $t \rightarrow \infty$. Uma vez que $\omega(x)$ é invariante, $V(\omega(x))=L$ implicando que $\dot{V}(\omega(x))=0$ e, assim, $\omega(x) \subset C$.

Como $\omega(x)$ é conexo, existe uma única componente conexa $C_{i}$, tal que $\omega(x) \subset \partial C_{i}$.

Considere o sistema (2) de tal forma que as hipóteses to Teorema 2.1 sejam satisfeitas.

Para cada $x \in \partial A(\sigma)$, seja $\left\{C_{j}, j \in \Lambda_{x}\right\}$ o conjunto de todos as componentes conexas de $C$ tais que $C_{j} \cap \partial A(\sigma) \neq \varnothing$ e $x \in \mathcal{W}^{s}\left(C_{j}\right)$. Pelo Teorema 2.1, $\Lambda_{x} \neq \varnothing$.

Para cada $j \in \Lambda_{x}$, ou $\omega(x) \cap C_{j} \neq \varnothing$ ou $\Phi^{+}(x) \cap C_{j} \neq \varnothing$.

Se $\Phi^{+}(x) \cap C_{j} \neq \varnothing$, seja $t_{j}=\min \left\{t>0: \Phi(t, x) \in C_{j}\right\}$ e defina o conjunto de controle relativo a $x$ como sendo a componente $C_{j}$ para a qual o menor $t_{j}$ ocorra. No caso em que $\Phi^{+}(x) \cap C_{j}=\varnothing$, pelo Teorema 2.2, $\Lambda_{x}$ é um conjunto unitário. Então, o único $C_{j}$ com $j \in \Lambda_{x}$ é definido como o conjunto de controle relativo a $x$.

Denotaremos por $C_{x}$ o conjunto de controle relativo ao ponto $x$.

Vejamos a definição de parte relevante da fronteira de estabilidade neste contexto.

Definição 2.2. A parte relevante da fronteira da região de estabilidade é o conjunto dos pontos de $\partial A(\sigma)$ para os quais o conjunto de controle é o mesmo do exit-point.

Considere $\bar{x} \in \partial A(\sigma)$ e defina o valor crítico de energia generalizada por

$$
K_{\bar{x}}=\min _{x \in \partial C_{\bar{x}}} V(x)
$$

Note que $V(\bar{x}) \geq K_{\bar{x}}$ para todo $\bar{x} \in \partial A(\sigma)$.

\section{Algoritmo Conceitual}

Para que uma estimativa da parte relevante da fronteira da região de estabilidade possa ser obtida com base nos resultados apresentados, é necessária uma forma de detectar quando a trajetória do sistema perturbado se aproxima da fronteira da região de estabilidade. 
A dificuldade em se realizar tal detecção também é encontrada no método CUEP, onde é superada através de métodos computacionais (ver $[5,6]$ ) que fornecem uma estimativa preliminar para a fronteira.

O leitor pode se perguntar: por que aplicar um método para estimar uma parte da fronteira da região de estabilidade quando, a priori, se precisa de uma estimativa preliminar? A resposta está nas vantagens em se obter uma estimativa conservadora. Através dela, é possível garantir se o sistema é capaz de suportar à perturbação.

Uma classe importante de sistemas em que tal estimativa inicial pode ser encontrada, é a dos sistemas quase-hamiltonianos. Neste caso, as curvas de nível da função hamiltoniana associada podem ser usadas para fornecer a estimativa inicial necessária. Ver seção 4.

Baseado nos resultados da seção 2, o seguinte algoritmo pode ser aplicado para se obter o tempo máximo em que uma perturbação pode ser aplicada a um sistema de tal forma que ele a suporte.

1. Encontre as componentes conexas do conjunto $C$;

2. Integre o sistema perturbado até que a solução se aproxime da estimativa inicial da fronteira de estabilidade do conjunto atrativo $\sigma$. Defina este ponto como $\bar{x}$;

3. Integre o sistema pós-perturbação até que a solução toque ou esteja suficientemente próxima de algum $C_{i}$. Defina este conjunto como o conjunto de controle $C_{\bar{x}}$.

4. Encontre o menor valor de energia na fronteira de $C_{\bar{x}}$ e defina o valor de energia crítica $K_{\bar{x}}$.

5. Se a $V(\sigma)<K_{\bar{x}}$, então a curva de nível $V(x)=K_{\bar{x}}$ é uma estimativa para a parte relevante da fronteira da região de estabilidade e o primeiro instante para o qual a trajetória do sistema perturbado intersecta esta curva de nível fornece uma estimativa conservadora para o tempo crítico $t_{c}$.

\section{Exemplo}

Considere a função $H(x, y)=y^{3}-y+x^{2}+y^{2}$ e o sistema (2) com

$$
f(x, y)=\left(\frac{\partial H}{\partial y},-\frac{\partial H}{\partial x}-0.1\left(y^{2}+1\right)\right)=\left(3 y^{2}+2 y-1,-2 x-0.1\left(y^{2}+1\right)\right),
$$

$\mathrm{e}$

$$
\tilde{f}(x, y)=\left(3 y^{2}+2 y-1,-0.1\left(y^{2}+1\right)\right) .
$$

O retrato de fase do sistema pós-perturbação é mostrado na Figura 1. Os pontos $p_{0}=(-1 / 18,1 / 3)$ e $p_{1}=(-1 / 10,-1)$ são, respectivamente, um ponto de equilíbrio assintoticamente estável e um ponto de equilíbrio do tipo sela.

Suponha que o sistema (2) esteja em estado de equilíbrio, correspondente ao ponto $p_{0}$, quando a perturbação ocorre.

Como o sistema pós-perturbação é quase-hamiltoniano, o conjunto de nível da função $H$ ao qual pertence o ponto crítico $x_{1}=(0,-1)$ fornece uma estimativa para a fronteira 


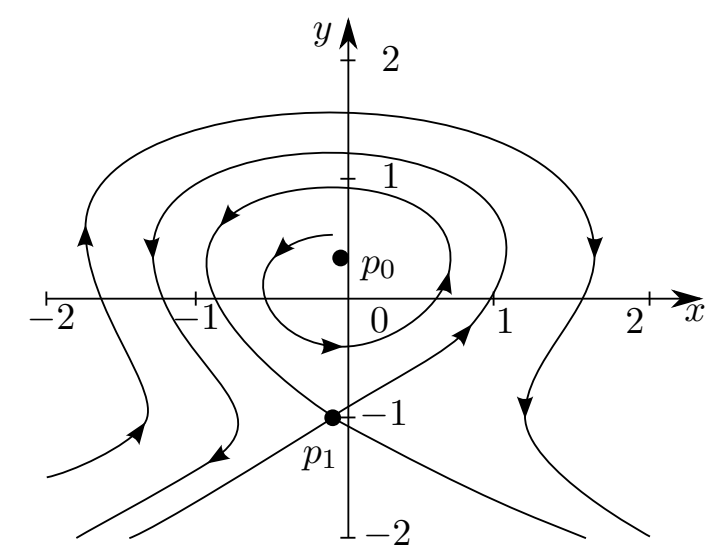

Figura 1: Retrato de fase do sistema quase-hamiltoniano definido por (4) e (5).

de estabilidade do ponto de equilíbrio assintoticamente estável $p_{0}$. Porém, a estimativa não é conservadora. A Figura 2 mostra como a estimativa obtida através da curva de nível $H(x, y)=H\left(x_{1}\right)$ se compara com a fronteira da região de estabilidade verdadeira.

Agora, de posse da seguinte FEG para o sistema pós-perturbação,

$$
\begin{aligned}
V(x, y) & =1.337 x-12.077 y+12.242 x^{2}-0.803 x y+12.396 y^{2}+0.683 x^{3}-0.443 x^{2} y \\
& +1.069 x y^{2}+11.323 y^{3}+0.109 x^{4}+1.111 x^{3} y+0.459 x^{2} y^{2}+1.7909 x y^{3} \\
& +0.064 y^{4}-0.270 x^{5}-1.467 y^{2} x^{3}+0.245 x^{2} y^{3}-1.135 y^{4} x+0.582 y^{5}
\end{aligned}
$$

podemos aplicar o algoritmo da seção 3 , obtendo $t_{c}=2,1$.

A Figura 2 mostra uma comparação entre a estimativa encontrada pelo algoritmo proposto e a região de estabilidade real. Note que, embora a estimativa seja relativamente menor que a região de estabilidade real, a estimativa é conservadora, o que não ocorre utilizando a função $H$.

\section{Conclusões}

O método apresentado se mostra bem sucedido na obtenção de estimativas conservadoras para partes relevantes da fronteira da região de estabilidade para sistemas dinâmicos que admitem FEG. Uma vez que a classe de sistemas que admitem FEG é mais ampla do que aquela que admite função energia, acredita-se que com o surgimento de métodos como o apresentado neste trabalho, técnicas bastante eficazes e eficientes para análise de estabilidade de sistemas não lineares se tornem disponíveis para sistemas com dinâmica mais complicada.

\section{Agradecimentos}

Os autores agradecem ao CNPq pelo suporte a este trabalho (número 305486/2013-6). 

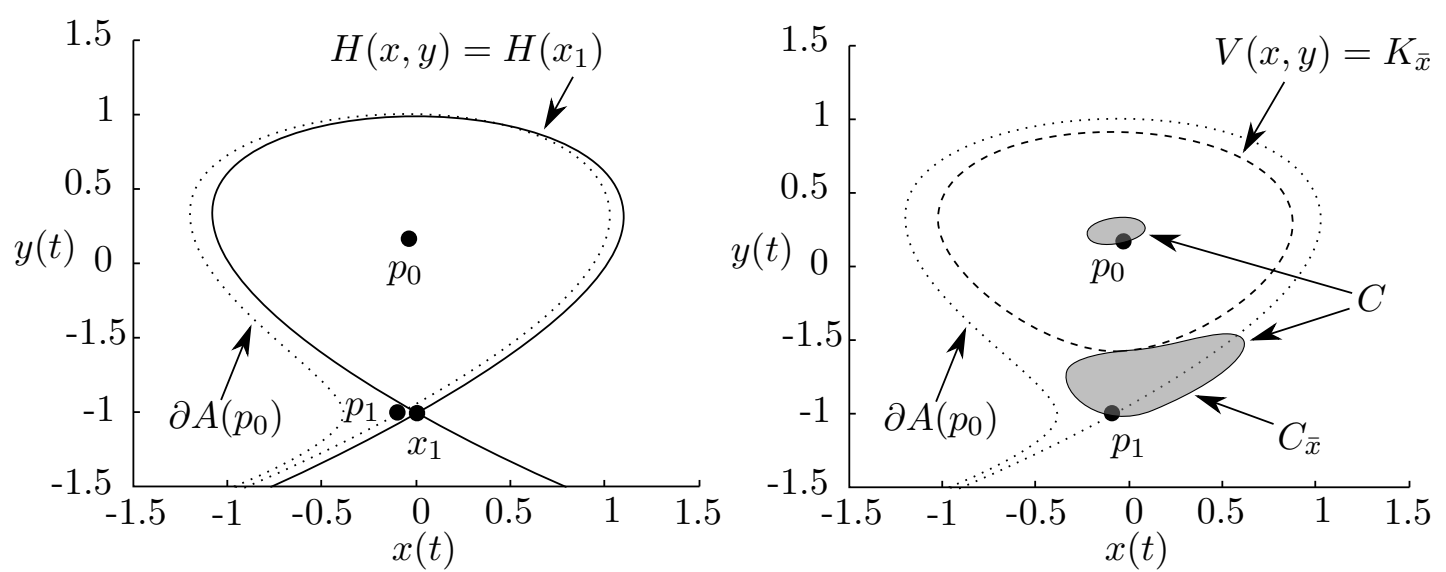

Figura 2: Comparação entre as estimativas obtidas através de um conjunto de nível de $H$ (à esquerda) e através do algoritmo proposto(à direita).

\section{Referências}

[1] L. F. C. Alberto and H.-D. Chiang. Characterization of stability region for general autonomous nonlinear dynamical systems. IEEE Transactions on Automatic Control, 57(6):1564-1569, 2012.

[2] L. F. C. Alberto and H.-D. Chiang. Towards development of generalized energy functions for electric power systems. IEEE Power and Energy Society General Meeting, pages 1-6, 2012.

[3] A. Chakraborty, P. Seiler, and G. J. Balas. Nonlinear region of attraction analysis for flight control verification and validation. Control Engineering Practice, 19(4):335-345, 2011.

[4] H.-D. Chiang. Study of the existence of energy functions for power systems with losses. IEEE Transactions on Circuits and Systems, 36(11):1423-1429, 1989.

[5] H.-D. Chiang. The bcu method for direct stability analysis of electric power systems: Theory and applications. IMA Volumes in Mathematics and Its Applications, 64:39-39, 1995.

[6] H.-D. Chiang. Direct Methods for Stability analysis of Electric Power Systems. Wiley, 2011.

[7] H.-D. Chiang, F. F. Wu, and P. P. Varaiya. Foundations of direct methods for power system transient stability analysis. IEEE Transactions on Circuits and Systems, 34(2):160-173, 1987.

[8] H. Hethcote, M. Zhien, and L. Shengbing. Effects of quarantine in six endemic models for infectious diseases. Mathematical Biosciences, 180:141-160, 2002. 